\section{Quantitative phase microscopy of articular chondrocyte dynamics by wide-field digital interferometry}

\author{
Natan T. Shaked, ${ }^{\mathrm{a}, *}$ John D. Finan, ${ }^{\text {a,b }}$ Farshid Guilak, ${ }^{\mathrm{a}, \mathrm{b}}$ \\ and Adam Wax ${ }^{\mathrm{a}}$ \\ ${ }^{a}$ Duke University, Department of Biomedical Engineering, \\ Fitzpatrick Institute for Photonics, Durham, North \\ Carolina 27708 \\ ${ }^{\mathrm{b}}$ Duke University Medical Center, Department of Surgery, \\ Durham, North Carolina 27710
}

\begin{abstract}
We experimentally implement label-free phase microscopy using wide-field digital interferometry (WFDI) techniques to retrieve quantitative volumetric data of articular chondrocyte dynamics. Using the scanless interferometric system, we visualize chondrocyte swelling and bursting induced by hypo-osmotic pressure. Reconstructed images are obtained by an efficient digital process. We use the resulting images to calculate quantitative temporal-spatial morphological parameters of the cell, with the observed dynamics limited only by the true frame rate of the camera. To show the utility of WFDI in recording articular chondrocyte dynamics, we also provide an experimental comparison of WFDI and differential interference contrast microscopy. @ 2010 Society of Photo-Optical Instrumentation Engineers. [DOI: 10.1117/1.3290242]
\end{abstract}

Keywords: interferometric microscopy; phase microscopy; cell imaging; chondrocytes; osmotic stress; cytology.

Paper 09461LR received Oct. 15, 2009; revised manuscript received Nov. 13, 2009; accepted for publication Nov. 24, 2009; published online Jan. 29, 2010.

Articular cartilage is the connective tissue that distributes mechanical loads between bones and provides almost frictionless surfaces in diarthrodial joints. Cartilage contains a single type of cells called chondrocytes. The phenotypic expression and metabolic activity of these cells are strongly influenced by shape and volume changes occurring due to mechanical and osmotic stresses. ${ }^{1}$ Due to the highly charged and hydrated nature of the cartilage extracellular matrix, mechanical loading causes an exudation of the interstitial water, resulting in dynamic changes in the interstitial osmolarity. Chondrocytes respond to osmotic stress by rapidly swelling or shrinking, followed by an active volume recovery response. Thus in vitro, instantaneous evaluation of the chondrocyte volumetric adaptation to such stresses can provide important information on the structure-function relationships in these cells.

Depending on the environmental stimuli, chondrocyte swelling can happen within a period of several seconds, with meaningful intermediate events that potentially occur within milliseconds. ${ }^{2}$ Important calculations that are associated with these rapid intermediate events include accurate volume

*Tel: 1-919-660-5588; E-mail: natan.shaked@duke.edu. evaluation at the time of maximal swelling (e.g., just before cell rupture), as well as dry-mass calculations of single cells and cell populations in monolayers during swelling, where other cells and intracellular parts rapidly move inside the field of view (FOV). Visualizing these rapid dynamic phenomena with the goal of obtaining quantitative and functional volumetric data requires microscopy techniques with fast 3-D acquisition rates that are beyond the scope of most scanning microscopy methods. Additionally, chondrocytes, like many other biological cells, are mostly transparent 3-D objects that are very similar to their surroundings in terms of absorbance and reflection, and thus typical light-intensity-based microscopy lacks the required contrast. In previous studies, the physicochemical (osmotic) properties of isolated chondrocytes in response to aniso-osmotic conditions have been measured using microscopy to determine changes in cell morphology and apparent membrane areas. ${ }^{1,3,4}$ The microscopy methods used include label-based methods such as fluorescence microscopy, ${ }^{3}$ as well as label-free methods such as phase contrast $(\mathrm{PhC})$ or differential interference contrast (DIC) microscopy. ${ }^{4}$ However, contrast agents such as fluorescent dyes have drawbacks, including potential cytotoxicity, the tendency to photobleach, and particularly for this case, a potential influence on the cell's response to osmotic changes. On the other hand, traditional phase microscopy methods such as $\mathrm{PhC}$ and DIC are not fully quantitative, and present artifacts that make it hard to convert the resulting images to quantitative measurements. In addition, these traditional methods require manual focus adjustment during the chondrocyte volume change, potentially distorting transient data. ${ }^{4}$

Digital interferometry, on the other hand, has the potential to provide a powerful noninvasive tool for quantitative phase measurements of 3-D, optically transparent biological cell dynamics. ${ }^{5-7}$ Using this approach, it is possible to obtain measurements at the nanometer scale of the optical phase delays of light transmitted through cells without special sample preparation or the use of contrast agents. By analyzing these real-time measurements, quantitative structural and dynamic cell information can be obtained. Transmission-mode digital interferometry requires the recording of an interference pattern composed of a superposition of the field transmitted through the sample and a mutually coherent reference field. Since the entire wavefront is captured, it is possible to reshape this wavefront and obtain 3-D pseudo-sectioning without mechanical scanning, or to use the captured wavefront to compensate for aberrations in the optical system. In wide-field digital interferometry (WFDI) with off-axis geometry, the entire wavefront is captured in a single camera exposure. This enables one to capture both the amplitude and the phase distributions of rapid 3-D dynamic phenomena. WFDI has been previously applied to quantitatively examine relatively slow osmotic-pressure-based transients in red blood cells, cancer cells, and kidney cells. 6,7

In this work, we apply quantitative phase analysis by WFDI to examine the dynamics of articular chondrocytes, which are a particularly interesting model system for studying physical signal transduction and cellular injury due to their sensitivity to physiologic changes in osmotic stress. ${ }^{8}$ Using WFDI, we visualize rapid chondrocyte swelling and bursting

1083-3668/2010/15(1)/010505/3/\$25.00 @ 2010 SPIE 


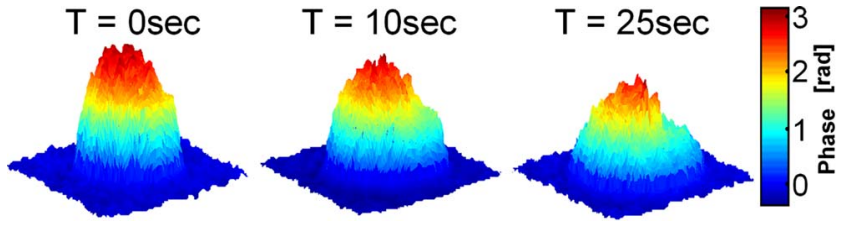

Fig. 1 Surface plots of the unwrapped phase profiles of chondrocyte dynamics obtained by WFDI.

due to osmotic changes. Based on the collected data, we quantitatively calculate the time dependence of the cell relative volume, dry mass, area, and average phase. We also provide an experimental comparison to DIC microscopy, a widely used label-free technique for chondrocyte measurements.

For this study, chondrocytes were isolated from articular cartilage of the lateral femoral condyles of 2- to 3-year-old skeletally mature female pigs as described in Refs. 1 and 2. Media osmolarity was increased to $380 \mathrm{mOsm}$ with sucrose, and verified with a freezing point osmometer (Precision Systems). Approximately $2 \times 10^{5}$ cells were seeded on each coverslip and cultured for 2 days. Prior to the experiment, the coverslip was mounted in a perfusion chamber (PeCon $\mathrm{GmbH}$ ) that was closed to prevent fluctuations of the media surface in the optical path. The chamber was initially loaded with approximately $400 \mu \mathrm{l}$ of serum-free media at 380 mOsm. During the experiment, rapid swelling was induced by adding 2 to $4 \mathrm{ml}$ of deionized water to the chamber using a syringe pump (Kent Scientific Corporation), yielding calculated final medium osmolality of 26 to $49 \mathrm{mOsm}$.

Transmission-mode Mach-Zehnder-based setup was used for recording the chondrocyte transient behavior during swelling due to osmotic pressure. Light from a laser source $(17 \mathrm{~mW}, 632.8-\mathrm{nm} \mathrm{HeNe})$ is split into reference and object beams. The object beam is transmitted through the sample and magnified by a microscope objective $(40 \times, \mathrm{NA}=0.66)$. The reference beam is transmitted through a compensating microscope objective, combined with the object beam, and both are projected, using a $15-\mathrm{cm}$ focal length lens, onto a digital camera [AVT PIKE F-032B, maximal full-frame rate 120 frames per second (fps)]. The object and reference beams are incident on the camera at an angle, creating an off-axis interferogram of the sample. Provided that there is a sufficient angle between the reference and object beams, ${ }^{9}$ a single interferogram can be used to retrieve both phase and amplitude of the sample field. Small changes in this angle during the measure-

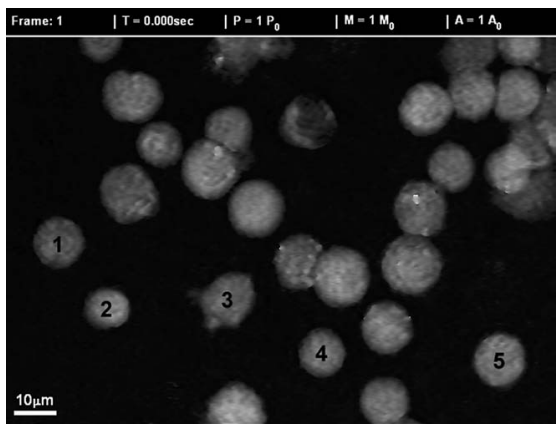

Video 1 Unwrapped phase profiles of chondrocyte monolayer dynamics obtained by WFDI (acquired at $120 \mathrm{fps}$, presented at $7.5 \mathrm{fps}$ ) (MPEG, 8.65 MB). [URL: http://dx.doi.org/10.1117/1.3290242.1]. ment of dynamic processes can cause potential error in the retrieved sample field. To avoid this issue, we digitally measure the carrier fringe frequency $\phi_{C}$ separately for each acquired interferogram. Assuming vertically aligned fringes, summing vertically the approximately flat-phase background interference around the sample yields a relatively noise-free sine wave, which then can be digitally fit to an ideal sine wave to estimate the ideal fringe frequency $\phi_{C}$. The method, which is similar in some sense to the procedure presented by Ferraro et al., ${ }^{10}$ works well when the sampled background interference area is large enough to digitally sense the fringe frequency. Practically, if the fringes are vertically straight, several pixel lines are enough for this purpose. Next, the acquired interferogram is multiplied by $\exp \left(j \phi_{C}\right)$ to center the useful-image term at the origin of the spatial-frequency domain. The remaining unwanted terms are spatially filtered by gradually discarding high spatial frequencies. The phase argument of the resulting complex matrix is the wrapped phase profile of the sample field, where an unwrapping algorithm removes $2 \pi$ ambiguities in this profile.

To demonstrate the utility of WFDI for visualizing chondrocyte dynamics, $120 \mathrm{fps}$ data were acquired during extreme osmolarity reduction. Figure 1 shows the surface plot of a single-chondrocyte phase profile during swelling at three chosen time points. As expected, during swelling, the phase profile height decreases and the 2-D area of the cell image increases. ${ }^{6}$ Video 1 presents a 2-D-view phase profile of chondrocyte monolayer dynamics during swelling, illustrating that chondrocyte swelling and bursting dynamics vary from cell to cell. The video was down-sampled to $7.5 \mathrm{fps}$ to reduce the file size. However, the quantitative analysis presented next was carried out at the full $120 \mathrm{fps}$ rate.

For comparison purposes, we have collected similar data using a line-scan DIC microscope (LSM 510, Carl Zeiss) at the maximum frame rate possible by this specific device (0.75 fps for cell-monolayer imaging), as shown in Video 2. Although the two experiments (DIC and WFDI) have been performed using separate systems, the same sample and imaging conditions were made as similar as possible. As expected from DIC microscopy, only the cell edges are seen, and a shading artifact is viewed. As also shown in this video, after several seconds the chondrocytes are no longer in focus. Since the DIC data collection is 2-D in nature, it is impossible to refocus the sample without losing important data during the transient. Thus, obtaining quantitative volumetric data at high frame rates is impractical using DIC microscopy.

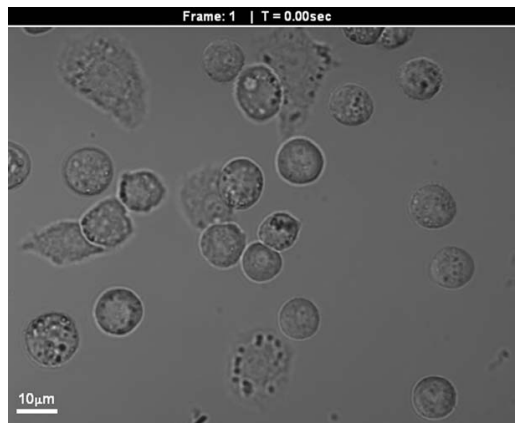

Video 2 Phase images of chondrocyte monolayer dynamics obtained by DIC microscopy (acquired and presented at $0.75 \mathrm{fps}$ ) (MPEG, 858.24 KB). [URL: http://dx.doi.org/10.1117/1.3290242.2]. 


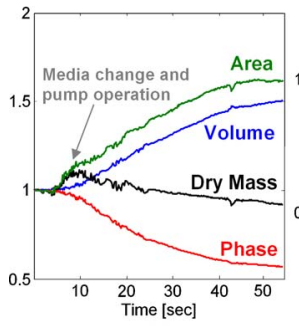

(a)

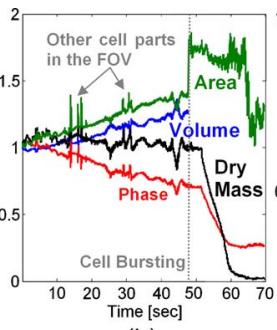

(b)

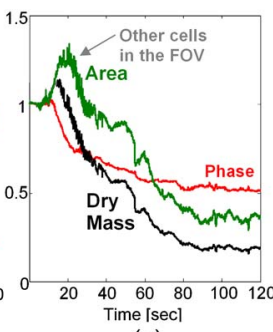

(c)
Fig. 2 WFDI-based graphs of the relative change in various chondrocyte morphological data during: (a) single-cell swelling (partially visualized in Fig. 1); (b) single-cell swelling and bursting; (c) cell monolayer dynamics (partially visualized in Video 1).

In our case, since biological cells are phase objects (and since WFDI captures the entire wavefront), we could correct this defocusing effect by digitally propagating along the axial direction to the transverse plane of the maximal phase value. ${ }^{11}$

The quantitative analysis performed on the final phase profiles obtained by WFDI includes ${ }^{6}$ monitoring the temporal changes in the chondrocyte relative area $\left(A / A_{0}\right)$, volume $\left(V / V_{0}\right)$, dry mass $\left(M / M_{0}\right)$, and average phase $\left(P / P_{0}\right)$. Figure 2(a) presents graphs of the temporal dependence of these parameters during the single-cell transient partially illustrated in Fig. 1. As can be seen from these graphs, the chondrocyte gained $46 \%$ in volume and $52 \%$ in area during swelling, while retaining its dry mass at approximately the same level. Figure 2(b) shows the parameter graphs for the transient of another single chondrocyte. In this case, the chondrocyte started swelling, gaining volume and area, but then burst and lost dry mass. This finding provides experimental support of the drymass calculations that are based on the chondrocyte phase profile. Another validation for this calculation is the small jumps that can be seen on the graphs before the chondrocyte bursts. These jumps exactly occur at the time points at which intercellular parts from other already-burst chondrocytes have entered the FOV. To our knowledge, this is the first time that dry-mass loss has been validated by induced cell bursting using WFDI. Note that the chondrocyte volume was calculated up until the time point of cell bursting (and not beyond) due to the fact that the volume calculation assumes isotropic volume change. ${ }^{6}$ Based on the high temporal resolution obtained, we calculate this chondrocyte volume just prior to bursting as $V_{L}=1.28$ times the initial cell volume $V_{0}$. Figure 2(c) shows graphs of the time dependence of the relative area, dry mass, and average phase of the cell monolayer visualized in Video 1. The graphs illustrate the tradeoffs in these parameters that occur during the dynamic response of the monolayer. Different chondrocytes start swelling at different time points, swell to various extents, and burst at different time points. Individual cell swelling and bursting result in a decrease in the average phase value. The rupture of an individual cell is characterized by a loss of dry mass and in a temporal increase of viewable area (until the burst chondrocyte intracellular parts leaves the FOV). On the other hand, other chondrocytes and chondrocyte intracellular parts entering the FOV result in an increase in the dry mass and area. As time passes, it is demonstrated that the values of all three parameters decrease due to rupture of most chondrocytes in the monolayer, which results in an approximately uniform distribution of the intracellular parts in the chamber. Using the monolayer WFDI results, we calculated individual chondrocyte maximum volume $V_{L}$ before cell rupture in response to the same monolayer osmotic conditions. For example, the chondrocytes marked by 1 through 5 in the Video 1 image have yielded $V_{L, 1}=1.58 V_{0}$, $V_{L, 2}=1.81 V_{0}, V_{L, 3}=1.37 V_{0}, V_{L, 4}=1.35 V_{0}$, and $V_{L, 5}=1.22 V_{0}$.

In conclusion, we have experimentally demonstrated quantitative analysis of chondrocytes by WFDI. We have obtained key morphological parameters, including the chondrocyte relative volume, dry mass, and area, based on a real-time visualization of the phase profile. We have also experimentally demonstrated the advantages of WFDI for quantitative analysis of chondrocytes, compared to the widely used DIC technique. The quantitative phase imaging methods demonstrated in this work provide a powerful research tool for observing the dynamics of articular chondrocytes and other types of biological cells, as well as for performing various cytological measurements in vitro including cell viability assays.

\section{Acknowledgments}

Supported by NSF grants BES-0348204 and CBET-0651622, as well as NIH grants AR50245, AR48182, and AG15768. Shaked greatly acknowledges the support of the Bikura Postdoctoral Fellowship from Israel.

\section{References}

1. F. Guilak, G. R. Erickson, and H. P. Ting-Beall, "The effects of osmotic stress on the viscoelastic and physical properties of articular chondrocytes," Biophys. J. 82(2), 720-727 (2002).

2. W. R. Trickey, F. P. T. Baaijens, T. A. Laursen, L. G. Alexopoulos, and F. Guilak, "Determination of the Poisson's ratio of the cell: recovery properties of chondrocytes after release from complete micropipette aspiration," J. Biomech. 39(1), 78-87 (2006).

3. F. Guilak, "Volume and surface area measurement of viable chondrocytes in situ using geometric modeling of serial confocal sections," $J$. Microsc. 173, 245-256 (1994).

4. L. G. Alexopoulos, G. R. Erickson, and F. Guilak, "A method for quantifying cell size from differential interference contrast images: validation and application to osmotically stressed chondrocytes," $J$. Microsc. 205, 125-135 (2002).

5. N. T. Shaked, M. T. Rinehart, and A. Wax, "Dual-interferencechannel quantitative-phase microscopy of live cell dynamics," Opt Lett. 34(6), 767-769 (2009).

6. G. Popescu, Y. Park, N. Lue, C. Best-Popescu, L. Deflores, R. R. Dasari, M. S. Feld, and K. Badizadegan, "Optical imaging of cell mass and growth dynamics," Am. J. Physiol.: Cell Physiol. 295(2), C538-C544 (2008).

7. M. Kemmler, M. Fratz, D. Giel, N. Saum, A. Brandenburg, and C. Hoffmann, "Noninvasive time-dependent cytometry monitoring by digital holography," J. Biomed. Opt. 12(6), 064002 (2007).

8. A. K. Amin, J. S. Huntley, P. G. Bush, A. Hamish, R. W. Simpson, and A. C. Hall, "Osmolarity influences chondrocyte death in wounded articular cartilage," J. Bone Jt. Surg., Am. Vol. 90, 15311542 (2008).

9. N. T. Shaked, Y. Zhu, M. T. Rinehart, and A. Wax, "Two-step-only phase-shifting interferometry with optimized detector bandwidth for microscopy of live cells," Opt. Express 17(18), 15585-15591 (2009).

10. P. Ferraro, S. De Nicola, A. Finizio, G. Coppola, S. Grilli, C. Magro, and G. Pierattini, "Compensation of the inherent wave front curvature in digital holographic coherent microscopy for quantitative phasecontrast imaging," Appl. Opt. 42, 1938-1946 (2003).

11. F. Dubois, C. Schockaert, N. Callens, and C. Yourassowsky, "Focus plane detection criteria in digital holography microscopy by amplitude analysis," Opt. Express 14, 5895-5908 (2006). 